\title{
A Novel Rotating HTS Flux Pump Incorporating a Ferromagnetic Circuit
}

\author{
Zhenan Jiang, Chirs W. Bumby, Rodney A. Badcock, Hae-Jin Sung, and Robert A. Slade
}

\begin{abstract}
High Temperature Superconductor (HTS) flux pumps enable large currents to be injected into a superconducting coil without requiring normal-conducting current leads. We present results from an experimental axial-type HTS rotating flux pump which employs a ferromagnetic circuit to focus incident flux upon a coated-conductor (CC) stator wire. We show that this device can inject currents of $>50 \mathrm{~A}$ into an HTS coil at $77 \mathrm{~K}$, and is capable of operating at flux gaps greater than $18 \mathrm{~mm}$. Accommodating a cryostat wall within this flux gap will enable future flux pump designs, in which all moving parts are located outside the cryostat.
\end{abstract}

Index Terms - Current leads, Ferromagnetic circuit, Flux pump, HTS coated conductor, YBCO.

\section{INTRODUCTION}

Quperconducting flux pump devices enable large-currents to $N_{\text {be injected into a superconducting coil, without the }}$ requirement for current leads which bridge between room temperature and the cryogenic environment [1-12]. As such, an efficient high- $T_{\mathrm{c}}$ superconducting (HTS) flux pump could substantially reduce the cryogenic heat load incurred when operating HTS coils and magnets. Hoffmann et al have previously reported a barrel-type rotating HTS flux pump, in which a series of permanent $\mathrm{Nd}-\mathrm{Fe}-\mathrm{B}$ magnets are arranged around the circumference of a cylindrical rotor [7]. As the rotor turns, the Nd-Fe-B magnets pass across a coated conductor (CC) stator wire so that magnetic flux traverses the wire and drives a transport current through a series-connected superconducting coil. The barrel-type HTS flux pump has been successfully used to energise an HTS NMR magnet [9], and several other authors have also reported this type of device [1012]. However, a key limitation of the barrel-type flux pump is that it requires that the major moving parts (i.e. the rotor and shaft bearings) are located inside the cryogenic envelope. This presents an additional heat load to the cryogenic system, as well as imposing significant design and maintenance challenges. A preferred arrangement would be to locate the rotor of the HTS flux pump at room temperature, whilst the superconducting circuit (including the flux pump stator wire) is placed inside the cryostat. Such a configuration would require that the flux gap between the rotor and stator is arranged to bridge across the

Manuscript submitted November 28, 2015. This work was supported by the New Zealand Ministry of Business, Innovation and Employment (MBIE) under contract no. RTVU1401.

Zhenan Jiang, Chris W. Bumby, and Rodney A. Badcock are with the Robinson Research Institute, Victoria University of Wellington, PO Box 33436, Lower Hutt 5046, New Zealand. (e-mail: zhenan.jiang@vuw.ac.nz). cryostat wall. However, the Hoffmann device will not operate at flux gaps of more than few millimetres - which is insufficient to accommodate an effective thermally-insulating cryostat wall [12]. We have recently shown that this limitation occurs because a DC voltage is only generated when magnetic flux can fully traverse the HTS stator wire without being blocked by a region of pinned flux $[12,13]$. This condition is met when the amplitude of the peak perpendicular field applied at the flux pump stator exceeds a threshold value which is equal to $2 B_{\mathrm{p}}$, where $B_{\mathrm{p}}$ is the effective penetration field of the coatedconductor stator wire $[14,15]$. For a typical $12 \mathrm{~mm}$ YBCO coated-conductor wire of $I_{\mathrm{c}}=310 \mathrm{~A}$, then $2 B_{\mathrm{p}} \sim 50 \mathrm{mT}$, and it is found to be necessary to place the flux pump stator wire within $\sim 7 \mathrm{~mm}$ of a $10 \mathrm{~mm}$ diameter Nd-Fe-B magnet in order to achieve fields which exceed this value.

Here, we report a novel prototype axial-type CC flux pump which is capable of operating at a much greater flux gaps than Hoffmann's barrel-type design. We have experimentally studied the DC behaviour of a closed superconducting circuit comprising this flux pump connected in series with an HTS coil $[10,12]$.

\section{EXPERIMENTAL METHOD}

Fig. 1 shows a schematic of our experimental device, which utilises ferromagnetic iron yokes (1020 steel) within the rotor and stator to form a magnetic circuit which focusses magnetic flux upon the HTS CC wire. This approach leads to a substantial increase in the applied perpendicular field at the stator wire, when compared to an isolated permanent magnet. The flux pump comprises an array of $9 \mathrm{Nd}-\mathrm{Fe}-\mathrm{B}$ permanent magnets $\left(\mathrm{N} 42,0.5^{\prime \prime} \times 0.5^{\prime \prime} \times 0.5^{\prime \prime}\right)$ equally distributed along the outer edge of the rotor yoke. The Nd-Fe-B permanent magnets used had a maximum energy product density (BHmax) of 42 MGOe (i.e. N42). The stator yoke has a set of protruding iron 'teeth' and a single HTS CC stator wire was located upon one of these teeth. A hole within the yoke allows the HTS CC stator wire to exit the high field region without being exposed to a magnetic field from the return path of the magnetic circuit. The iron 'teeth' on the yoke focus the applied magnetic flux from the rotor magnets. This maximises the local applied magnetic field as the rotor magnet passes over the 'tooth'. The CC has a self-

Hae-Jin Sung is with the Department of Electrical Engineering, Changwon National University, 9 Sarim-Dong, Changwon 641-733, Republic of Korea. 
field $I_{\mathrm{c} 0}$ of $313 \mathrm{~A}$, and was connected via normal-conducting soldered joints to the HTS coil, which has a measured selfinductance $L=2.4 \mathrm{mH}$ at $77 \mathrm{~K}$. A cryogenic Hall sensor was fixed at the centre of the HTS coil and used to measure the current within the superconducting circuit. The sensor and coil were calibrated using an external current supply, prior to connection of the coil to the flux pump. From previous measurements [10,12], the total resistance of the soldered joints in the closed superconducting circuit was estimated to be approximately $1 \mu \Omega$. The axial flux gap between the rotor magnets and the CC stator (denoted $g$, in Fig. 1(b)) was varied from $2.6 \mathrm{~mm}-18.8 \mathrm{~mm}$ through the use of a series of interchangeable fixed spacer plates. The rotor was driven by a servo motor via a shaft which extended to the room temperature environment. Both the rotor and stator were placed in a liquid nitrogen $\left(\mathrm{LN}_{2}\right)$ bath for most of the measurements. However, the rotor was above the $\mathrm{LN}_{2}$ level for the two largest gap measurements $(g=16.9 \mathrm{~mm}$ and $18.8 \mathrm{~mm}$ ) in order to reduce the turbulent drag on the rotor at high rotational speeds. The speed of the servo motor was controlled such that the passing frequency of the rotor magnets, $f$, could be adjusted between zero and $360 \mathrm{~Hz}$.

\section{FIG. 1 HERE}

\section{EXPERIMENTAL RESULTS AND DISCUSSION}

Fig. 1(c) compares the values of the maximum amplitude of the perpendicular magnetic field, $B_{\text {peak }}$, generated at the HTS stator wire by these magnets as a function of the flux gap, $g$ for two cases: (i) Our axial flux pump design utilising the ferromagnetic circuit; and (ii) the earlier-reported [10, 12] barrel-type flux pump employing isolated permanent magnets in the absence of a ferromagnetic circuit. The magnetic field values were calculated by the finite-element method using Opera software [16]. Fig. A1 shows the magnetic field distribution in the stator and rotor yoke pieces, which has been calculated using Opera software for $g=2 \mathrm{~mm}$. The magnetic field with MC at a flux gap, $g=2 \mathrm{~mm}$ is approximately four times of that without MC, and the axial-type device maintains a magnetic field amplitude of more than $50 \mathrm{mT}$ at flux gaps of more than $20 \mathrm{~mm}$. This shows that the magnetic circuit is highly effective at enhancing the amplitude of the magnetic field applied at large flux gaps.

Fig. 2(a) shows experimentally measured values of the injected coil current versus time, $t$, which were obtained when operating the flux pump at various flux gaps. Data at the operating frequency of $360 \mathrm{~Hz}$ (equivalent to $2400 \mathrm{rpm}$ ) was not available for small flux gaps, due to the large drag of $\mathrm{LN}_{2}$ upon the rotor at this speed. In all cases it took approximately $20 \mathrm{~s}$ for the heavy rotor iron yoke to accelerate to the target rotation speed. The coil current is observed to saturates at a value, $I_{\text {sat }}=I(t \rightarrow \infty)$, which occurs when the output voltage of the flux pump is equal to the resistive losses within the circuit. At the largest gap, $g=18.8 \mathrm{~mm}$, a coil current of $7.7 \mathrm{~A}$ had been injected after 1.2 hours. We observe some fluctuations in the injected current at this large gap value, which are probably caused by minor variations in the experimental conditions over the long time period of this experiment. These may be due to temperature fluctuations in the $\mathrm{LN}_{2}$ bath or mechanical vibrations of the rotor which lead to variation in the flux gap, $g$.

Fig. 2(b) shows data from the same experimental runs shown in Fig. 2(a) but now plotted as the instantaneous current, $I$, versus the instantaneous output voltage of the flux pump, $V_{\text {coil }}$, at each flux gap. We observe a straight line relationship between output current and voltage for all flux gaps and frequencies measured. This clearly demonstrates that the superconducting stator of the flux pump exhibits an effective internal resistance, $R_{\mathrm{d}}$, which is independent of the current in each case (as $R_{\mathrm{d}}=-\mathrm{d} V / \mathrm{d} I$ following the sign convention used in this plot). $R_{\mathrm{d}}$ is observed to change with varying flux gap and frequency, and hence cannot be attributed to the constant resistance of soldered joints within the circuit. This is consistent with previously reported results for barrel-type flux pumps [10, 12], where the internal resistance has been attributed to the effect of dynamic resistance within the HTS stator wire [17-19]. This effect occurs in a type-II superconductor carrying a DC transport current, whilst simultaneously experiencing an oscillating applied magnetic field. Dynamic resistance is a form of hysteretic loss, which means that the resulting effective resistance is proportional to frequency, and will increase with increasing amplitude of the applied field [17-19]. We observe both of these features in our experimental data.

\section{FIG. 2 HERE}

Based on the above observations, we can model the DC behaviour of the superconducting circuit using the simple equivalent circuit diagram $[10,12]$ shown in Fig. 3. Here, the flux pump is considered to be a DC voltage source with an open circuit voltage $V_{\text {oc }}$ and an effective internal resistance, $R_{\mathrm{d}}$. $V_{\mathrm{oc}}$ is proportional to the rate at which flux cuts the HTS stator, which is determined by the rotational speed of the flux pump rotor. The superconducting coil has an inductance, $L$, and the governing equation for the circuit is,

$$
L \frac{d I}{d t}+I R=V_{\mathrm{oc}}
$$

where the total resistance is given by $R=R_{\mathrm{c}}+R_{\mathrm{d}}$, with $R_{\mathrm{c}}$ denoting the contact resistance of soldered joints within the HTS circuit, and $R_{\mathrm{d}}$ denoting the dynamic resistance experienced at the flux pump stator, can be expressed as [10, $12,18]$,

$$
R_{\mathrm{d}}=\frac{4 a l f}{I_{\mathrm{c} 0}}\left(B_{\mathrm{a}}+\frac{B_{\mathrm{a}}{ }^{2}}{B_{0}}\right)
$$

where $2 a$ is the width of the stator CC and $l$ is the interaction length of the AC magnetic field with the conductor. $I_{\mathrm{c} 0}$ and $B_{0}$ are parameters from the Kim model [20] for critical current, 
$I_{\mathrm{c}}=I_{\mathrm{c} 0} /\left(1+B_{\mathrm{a}} / B_{0}\right)$ and $B_{\mathrm{a}}$ is the amplitude of the time-varying magnetic field. $L$ is the coil inductance, $V_{\mathrm{oc}}$ is the open-circuit voltage. We can solve for the current, $I$, as a function of time, $t$, to obtain

$$
I(t)=\frac{V_{\mathrm{oc}}}{R}\left(1-e^{-\frac{R}{L} t}\right)=I_{\mathrm{sat}}\left(1-e^{-\frac{R}{L} t}\right)
$$

where $I_{\mathrm{sat}}=V_{\mathrm{oc}} / R$ is the limiting saturation current, which is the maximum current that can be delivered by the flux pump. Similarly, the voltage across the coil is given by,

$$
V_{\text {coil }}(t)=L \frac{d I}{d t}=V_{\text {oc }} \cdot e^{-\frac{R}{L} t}
$$

From Eqs. (3) and (4) we see that the time constant during flux pump operation, $\tau=L / R$. From Fig. 2(b) we find that $R_{\mathrm{d}}$ is much larger than $R_{\mathrm{c}}(\approx 1 \mu \Omega)$, under all of the operating conditions measured here. From Eq. (3) we see that in this case, the contact resistance of soldered joints within our circuit plays no role in determining the limiting current delivered by the HTS flux pump, as $R \approx R_{\mathrm{d}}$ and hence $I_{\mathrm{sat}} \approx I_{\mathrm{sc}}=V_{\mathrm{oc}} / R_{\mathrm{d}}$, where $I_{\mathrm{sc}}$ is the short-circuit current which ignores contact resistance $R_{\mathrm{c}}$. Therefore, it is the inherent internal resistance, $R_{\mathrm{d}}$, that sets a limit for the maximum achievable current that can be delivered by this type of flux pump [10, 12]. It should be noted that if the $I_{\mathrm{sc}} \geq I_{\mathrm{c}, \text { coil }}$ this situation does not hold - as in this case the limiting current is determined by the point at which the output voltage of the flux pump matches the voltage dropped across the coil. This is clearly illustrated in Fig. 2(b) which shows that for $g=$ $2.6 \mathrm{~mm}$ and $5.8 \mathrm{~mm}$, the experimental range of output data from the flux pump is terminated by the $I-V$ curve of the HTS coil.

\section{FIG. 3 HERE}

Fig. 4 shows the output voltage of the flux pump (measured across the coil) as a function of time. After the initial acceleration time of $\sim 20 \mathrm{~s}$, the output voltage obtained at each flux gap exhibits the expected exponential decay described by Eq. (4). At $g=2.6 \mathrm{~mm}$ we observe the added factor that over a long time interval the voltage saturates at a value determined by the $I-V$ curve of the coil. The time constant, $\tau=L / R$, can be obtained from the initial gradient of each line in Fig. 4. We observe that $\tau$ increases as the flux gap increases, which is a result of a decreasing value $R_{\mathrm{d}}$. The voltage at zero time $V(t=$ $0)=V_{\mathrm{oc}}$, can be obtained from the intercept between the vertical axis and the straight line portion of each decay curve, and is consistent with the values obtained from Fig. 2(b).

\section{FIG. 4 HERE}

Fig. 5 shows values for the open-circuit voltage, $V_{\mathrm{oc}}$ and shortcircuit current, $I_{\mathrm{sc}}$ and internal resistance $R_{\mathrm{d}}$, which have been extracted from Fig. 2(b) (from the intercepts on the $V$ and $I$ axes and the gradient of each line respectively). This data is plotted as a function of flux gap, $g$. Fig. 5(a) compares the obtained $I_{\mathrm{sc}}$ values between our axial-type flux pump and the previously reported barrel flux pump [12] without magnetic circuit as a function of flux gap. It is clear that a substantial increase in $I_{\mathrm{sc}}$ is achieved by employing the ferromagnetic circuit.

Figs. 5(b) and (c) show plots of the values of $V_{\mathrm{oc}}$ and $R_{\mathrm{d}}$ after these values have been normalized by the frequency, $f$. Values obtained for $V_{\mathrm{oc}} / f$ and $R_{\mathrm{d}} / f$ at different rotor speeds approximately agree with each other, which confirms the expected result that $V_{\mathrm{oc}}$ and $R_{\mathrm{d}}$ are both linearly proportional to frequency [10,12]. $V_{\mathrm{oc}}$ and $R_{\mathrm{d}}$ both decrease as the flux gap increases, which is a direct result of the decrease in the applied magnetic field as the rotor magnet moves further away from the HTS stator CC. It can be seen that $V_{\text {oc }}$ decreases more rapidly with increasing $g$ than is the case for $R_{\mathrm{d}}$. This is consistent with the observed decrease in $I_{\mathrm{sc}}\left(=V_{\mathrm{oc}} / R_{\mathrm{d}}\right)$ with increasing flux gap, which is shown in Fig. 5(a). Results obtained at $360 \mathrm{~Hz}$ show that $V_{\text {od }} / f$ approaches zero for $g \geq 18.8 \mathrm{~mm}$, and we were unable to measure pumped currents at a flux gap larger than this value. As this is a frequency-normalised value, this is expected to be the case regardless of whether higher rotor speeds could be employed. In all cases, the frequency-normalized values of both $V_{\mathrm{oc}}$ and $R_{\mathrm{d}}$ are much larger than those previously reported for the barrel-type flux pump [12]. This is a direct result of the increased amplitude of the applied magnetic field at the CC stator, arising from the presence of the ferromagnetic circuit in this device.

\section{FIG. 5 HERE}

Fig. 6 shows the normalised open circuit voltage, $V_{\text {oc }} / f$ as a function of the calculated maximum applied perpendicular magnetic field experienced at the stator wire. This figure shows that $V_{\text {ocd }} f$ decreases approximately linearly with the amplitude of the peak applied field, and drops to approximately zero at a value slightly less than $67 \mathrm{mT}$ (at $g>18.8 \mathrm{~mm}$ ). This is broadly consistent with the calculated threshold value of $2 B_{\mathrm{p}} \approx 50 \mathrm{mT}$ which has previously been identified for the barrel-type device [12], and again indicates that the introduction of the ferromagnetic circuit has not changed the underlying operating principles of the rotating HTS flux pump.

\section{FIG. 6 HERE}

A key difference between the barrel-type flux pump and the axial-type device reported here is the presence of the iron yoke. This will lead to an additional parasitic heat load within the cryogenic environment caused by eddy currents and ferromagnetic hysteresis within the iron. It is well established that eddy-current loss can be largely eliminated through the use of a laminated structure. However, iron hysteresis loss is an inherent feature of this flux pump design. At $g=8.6 \mathrm{~mm}$ and $f$ $=180 \mathrm{~Hz}$ the stator experiences a peak-to-peak $B$-field amplitude of $217 \mathrm{mT}$ (see Fig. 6). Under these conditions, we estimate a highly conservative upper limit for the heat load due to iron hysteresis is approximately $80 \mathrm{~mW}$ [21]. This is a substantial over-estimation as it assumes that all parts of the 
iron yoke experience the full oscillating field amplitude. For comparison, the estimated heat load from a pair of current leads carrying $35 \mathrm{~A}$ bridging $300 \mathrm{~K}$ and $77 \mathrm{~K}$ is $1.4 \mathrm{~W}$ [22]. This is far larger than the estimated upper limit for the ferromagnetic hysteresis loss.

\section{CONCLUSION}

We have demonstrated a novel axial-type coated conductor rotating flux pump which can inject current into an HTS coil at flux gaps of up to $18.8 \mathrm{~mm}$. This device utilises a magnetic circuit to focus magnetic flux upon the coated-conductor stator. We have shown that this device can be described by the same simple circuit model which has previously been used to describe barrel-type flux pumps, where the internal resistance and DC open-circuit are proportional to frequency. There are significant implications of this work for the application of flux pump technology to HTS magnet systems. A flux gap of 18.8 mm provides ample space to accommodate a thermally efficient and mechanically sound cryostat wall. In this manner it is now possible to design a flux pump in which the flux pump rotor is entirely located at room temperature whilst the superconducting stator remains inside the cryostat. This approach completely eliminates the requirements for penetration of the cryostat wall by current injecting metal leads, and presents the possibility of designing highly thermally efficient cryogenic magnet systems. We are now actively investigating the application of such a device to an HTS generator, where an externally located flux pump can act as a "brushless exciter" for the HTS rotor coils.

\section{APPENDIX}

\section{FIG. A1 HERE}

\section{REFERENCES}

[1] H. van Bleelen, A. J. P. T. Arnold, H. A. Sypkens, J. P. van Braam Houckgeest, R. De Bruyn Ouboter, J. J. M. Beenakker, and K. W. Taconis, "Flux pumps and superconducting solenoids, " Physica, vol. 31, pp. 413-443, 1965.

[2] O. K. Mawardi, A. Gattozzi, and H. Chung, "Operational characteristics of a flux pump, ”IEEE Trans. Magn., vol. 15, pp. 828-831, Jan. 1979.

[3] L. J. M. van de Klundert and H H J ten Kate, "Fully superconducting rectifiers and flux pumps Part 1: Realized methods for pumping flux," Cryoginics, vol. 21, pp. 195-206, Apr. 1981.

[4] L. J. M. van de Klundert and H H J ten Kate, "On fully superconducting rectifiers and fluxpumps. A review. Part 2: Commutation modes, characteristics and switches," Cryoginics, vol. 21, pp. 267-277, May, 1981.

[5] S. Ishmael, C. Goodzeit, P. Masson, R. Meinke, and R. Sullivan, "Flux pump excited double-helix rotor for use in synchronous machines, "IEEE Trans. Appl. Supercond., vol. 18, pp. 693-696, June 2008.

[6] T. Nakamura, M. Sugano, T. Doi, and N. Amemiya, "Flux pump effect of HTS films in a traveling magnetic field," IEEE Trans. Appl. Supercond., vol. 20, pp. 1033-1036, June 2010.

[7] C. Hoffmann, D. Pooke, and A. D. Caplin, "Flux pump for HTS magnets," IEEE Trans. Appl. Supercond., vol. 21, pp. 1628-1631, June 2011.

[8] Z. Bai, C. Chen, Y. Wu, and Z. Zhen, "Effect of various pulse wave forms for pulse-type magnetic flux pump," Cryogenics, vol. 51, pp. 530-533, July 2011.

[9] R. M. Walsh, R. Slade, P. Donald, and C. Hoffmann, "Characterization of current stability in an HTS NMR system energized by an HTS flux pump," IEEE Trans. Appl. Supercond., vol. 24, June 2014, Art. ID 4600805 .
[10] Z. Jiang, K. Hamilton, N. Amemiya, R. A. Badcock, and C. W. Bumby, "Dynamic resistance of a high- $T_{\mathrm{c}}$ superconducting flux pump," Appl. Phys. Lett, vol. 105, Sep. 2014, Art. ID 112601.

[11] T. A. Coombs, J. F. Fagnard, and K. Matsuda, "Magnetization of 2-G coils and artificial bulks," IEEE Trans. Appl. Supercond., vol. 24, Oct. 2014, Art. ID 8201005.

[12] Z. Jiang, C. W. Bumby, R. A. Badcock, H. J. Sung, N. J. Long, and N. Amemiya, "Impact of flux gap upon dynamic resistance of a rotating HTS flux pump," Supercond. Sci. Technol., vol. 28, Sep. 2015, Art. ID 115008.

[13] C. P. Poole, H. A. Farach, and R. J. Creswick Superconductivity, Academic Press: New York, 1995, pp. 379-383.

[14] M. Iwakuma, K. Toyota, M. Nigo, T. Kiss, K. Funaki, Y. Iijima, T. Saitoh, Y. Yamada, and Y. Shiohara, "AC loss properties of YBCO superconducting tapes fabricated by IBAD-PLD technique," Physica C, vol. 412-414, pp. 983-991, May 2004.

[15] E. H. Brandt and M. Indenbom, "Type-II-superconductor strip with current in a perpendicular magnetic field," Phys. Rew. B, vol. 48, pp. 12893-12906, Nov. 1993.

[16] http://operafea.com/

[17] K. Ogasawara, K. Yasukochi, S. Nose, and H. Sekizawa, "Effective resistance of current-carrying superconducting wire in oscillating magnetic fields 1: Single core composite conductor," Cryoginics, vol. 16, pp. 33-38, Jan. 1976.

[18] M. P. Oomen, J. Rieger, M. Leghissa, B. ten Haken, and H. H. J. ten Kate, "Dynamic resistance in a slab-like superconductor with $J_{\mathrm{c}}(B)$ dependence," Supercond. Sci. Technol., vol. 12, pp. 382-387, 1999.

[19] R.C. Duckworth, Y. F. Zhang, T. Ha, and M. J. Gouge "Dynamic resistance of YBCO-coated conductors in applied AC fields with DC transport currents and DC background fields," IEEE Trans. Appl. Supercond., vol. 21, pp. 3251-3256, June 2011.

[20] Y. B. Kim, C. F. Hempstead and A. R. Strnad "Critical persistent currents in hard superconductors," Phys. Rev. Lett., vol. 9, pp. 306-309, 1962.

[21] http://www.protolam.com/

[22] S. S. Kalsi Applications of High Temperature Superconductors to Electric Power Equipment, IEEE Press: New Jersey, 2011, pp. 53. 
(a)
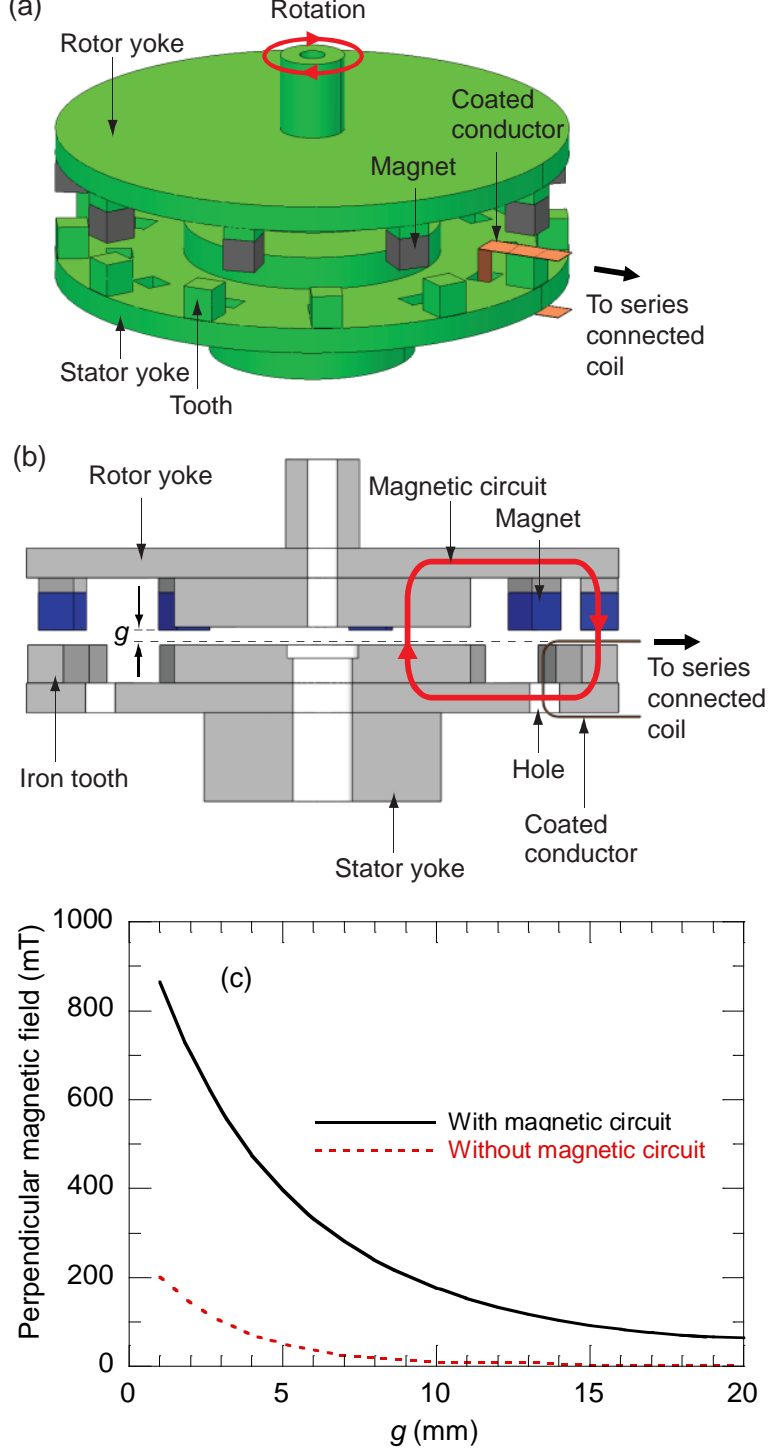

Fig. 1. (a) 3D view of the axial-type HTS flux pump reported in this work. (b) Cross-section diagram of the flux pump showing magnetic circuit formed between rotor and stator yokes. (c) Plot showing the maximum amplitude of the applied perpendicular magnetic field, Bpeak, at the HTS stator conductor versus flux gap, g. Calculated values shown for (i) the axial flux pump with magnetic circuit; and (ii) the previously reported barrel-type flux pump without magnetic circuit.
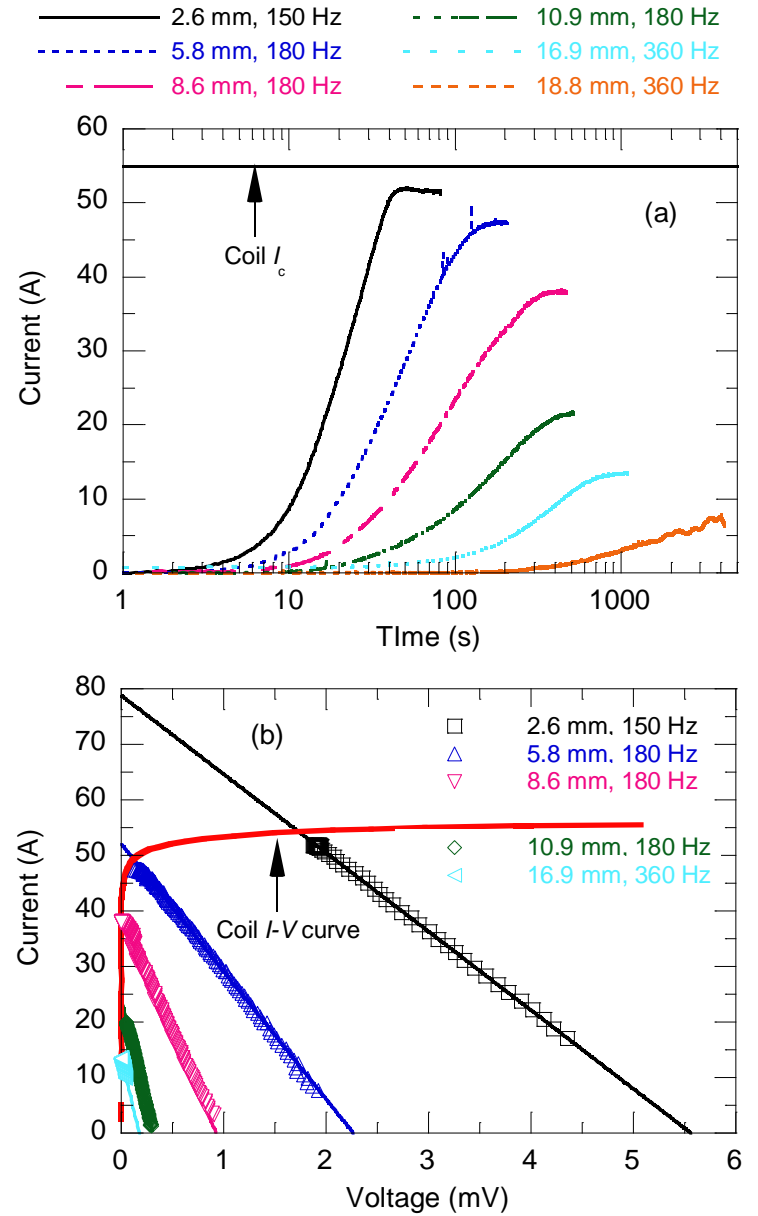

Fig. 2. (a) Comparison of injected coil currents versus time, $t$, for various flux gaps and operating frequencies. (b) Comparison of $V-I$ relationships obtained from the flux pump at various flux gaps.

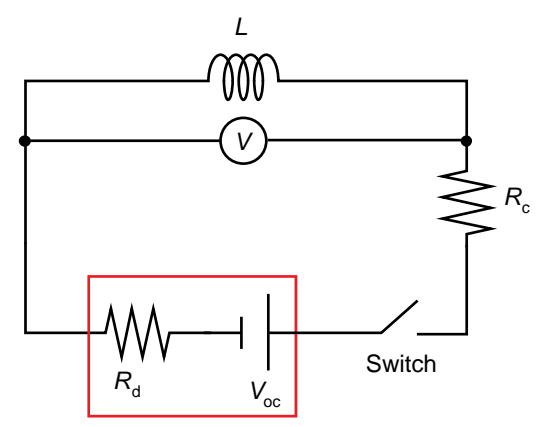

Flux pump

Fig. 3. Equivalent circuit diagram of a superconducting circuit comprising an HTS flux pump and an HTS coil of inductance, $L$. The flux pump has an internal resistance of $R_{\mathrm{d}}$, and outputs an open-circuit voltage, $V_{\mathrm{oc}} . R_{\mathrm{c}}$ denotes the total circuit resistance of the HTS circuit due to soldered joints between the HTS wires and the $I-V$ relationship of the coil (when $I(t)>I_{\mathrm{c}, \text { coil }}$ ). 


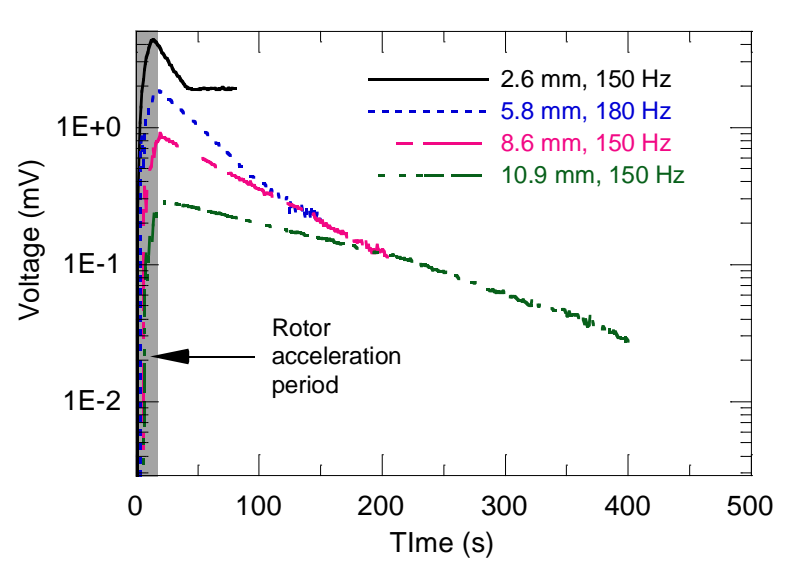

Fig. 4. Comparison of DC output voltages from the flux pump versus time, $t$, for various flux gaps and operating frequencies.
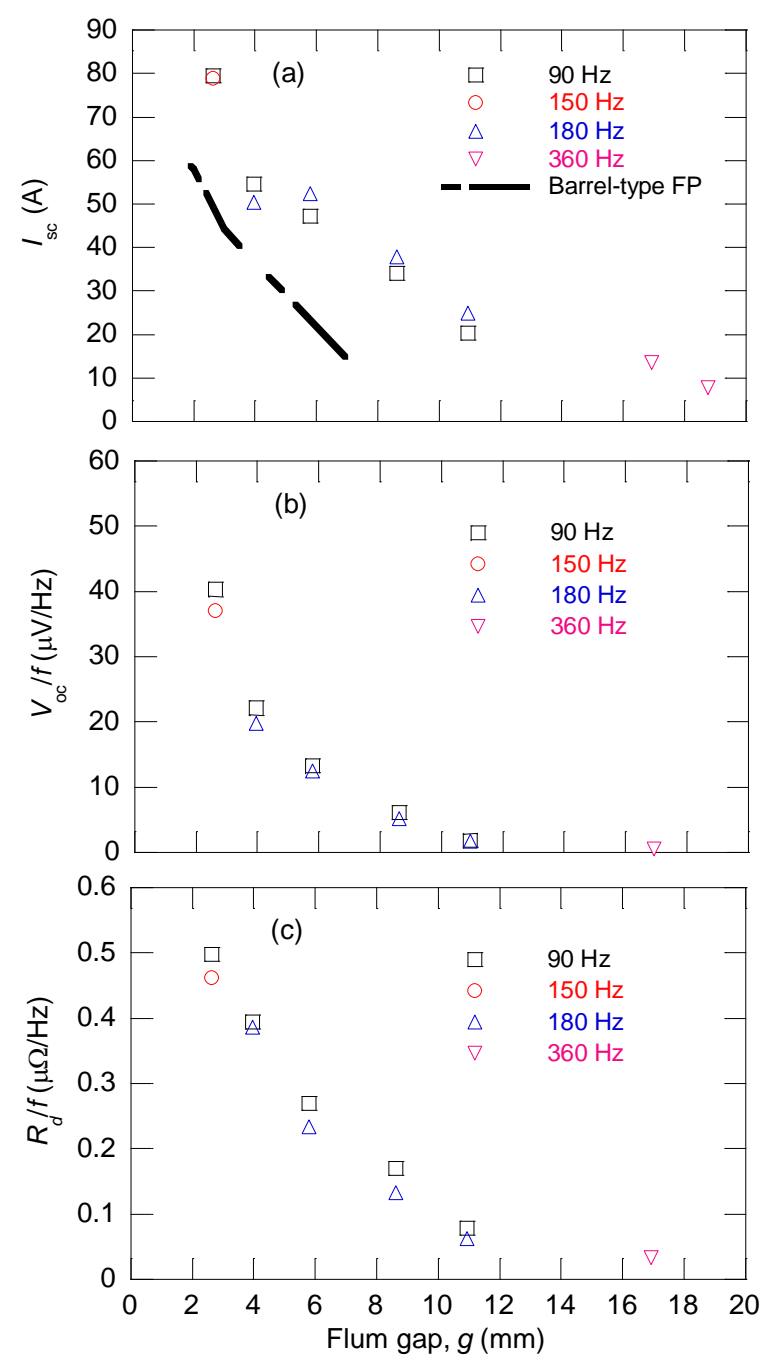

Fig. 5. (a) Values of the short-circuit current $I_{\mathrm{sc}}$, obtained from the axial-type flux pump versus flux gap, $g$. Also plotted for comparison are previouslyreported values10) of $I_{\mathrm{sc}}$ obtained from the barrel-type flux pump using similar stator wire and rotor magnets.(b) Frequency-normalized values for the opencircuit voltage, $V_{\text {oc }}$ versus flux gap. (c) Frequency-normalized values for the internal resistance, $R_{\mathrm{d}}$ of the flux pump versus flux gap.

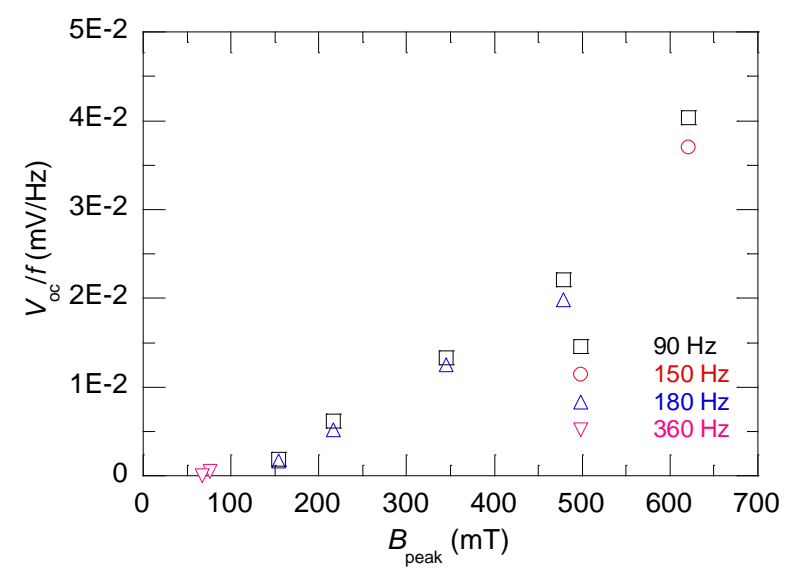

Fig. 6. Frequency-normalized open-circuit voltage $V_{\text {oc }}$ plotted as a function of the maximum applied perpendicular magnetic field at the stator, $B_{\text {peak }}$.

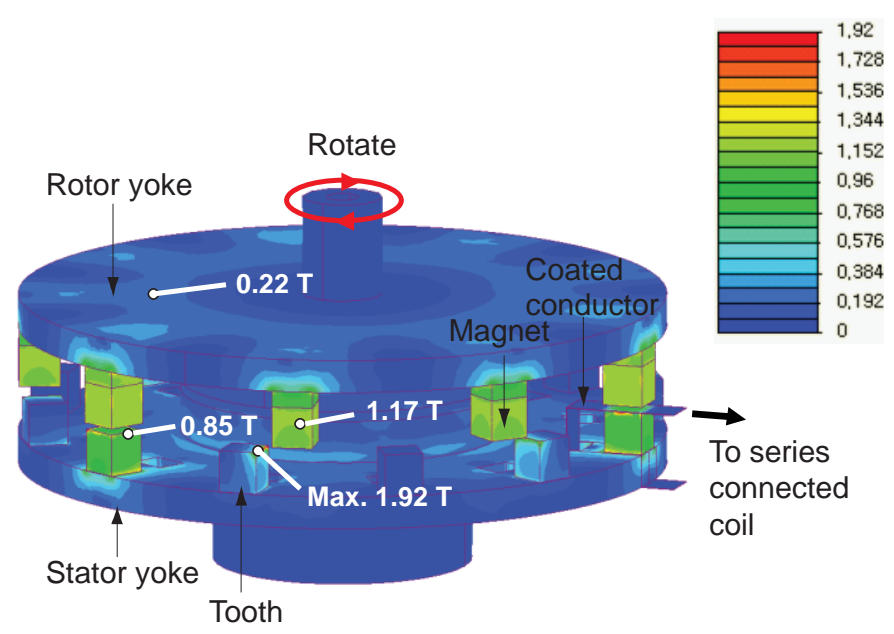

Fig. A1. Example of magnetic field distribution in the stator and rotor yoke pieces in the flux pump calculated using Opera software for $g=2 \mathrm{~mm}$. 
Zhenan Jiang received a B.Eng. in Electrical Engineering from Chongqing University in Chongqing, China in 1994, and M.Eng., Ph.D. Eng. in applied superconductivity from Yokohama National University in Yokohama, Japan in 2002 and 2005, respectively.

He was a Postdoctoral Research Fellow at Yokohama National University from 2005 to 2008. As a Research Scientist, he joined the Superconductivity Group currently known as the Robinson Research Institute at Victoria University of Wellington, New Zealand in 2008. He is now Senior Scientist at the same institute. He has a strong track record in characterization of high temperature superconductors (HTS), especially in AC loss and has first-authored more than 25 international journal papers. His recent research interests include AC loss characterization in HTS coils, HTS flux pumps, and rotating machines.

Dr. Jiang has been awarded a Japanese Government (MEXT) Scholarship for doctoral study (2002-2005), and twice awarded the JSPS (Japan Society for the Promotion of Science) invitation fellowship to Kyoto University under the NZ-Japan Scientist Exchange Program, in 2011 and 2015, respectively.

Chris W. Bumby is obtained his MPhys degree in Physics in 2000 from the University of Oxford, UK, and he received his DPhil in condensed matter physics from the same institution in 2005.

From 2005 to 2010 he was a Postdoctoral Research Fellow at the MacDiarmid Institute at Victoria University of Wellington. In 2011 he was appointed as a Research Scientist in the Superconductivity Research Group at Industrial Research Limited (IRL). In 2014 he became a founding member of the Robinson Research Institute at Victoria University of Wellington, New Zealand where he now holds the position of Senior Scientist.

Dr. Bumby has previously been awarded a Royal Society (UK) International Postdoctoral Fellowship (2005-2006), the Foundation of Research Science and Technology (FRST) Research Fellowship (2008-2010) and the IPENZ Ray Meyer Medal (2013).

Rodney A. Badcock was born in Cambridge, UK, in 1969. He received a B.Sc in Physics with Electronics from University of Leeds, and M.Sc. and Ph.D. degrees in manufacturing and materials engineering from Brunel University.

$\mathrm{He}$ has 25 years research experience in applied $\mathrm{R} \& \mathrm{D}$ covering manufacturing process monitoring and control, materials sensing and superconducting systems. His experience covers work at Royal Aerospace Establishment (UK), growing a fiber sensing startup into full production (Protodel International), through to Senior Lecturer in Engineering Systems at Cranfield University (UK). Since 2006 he has concentrated on superconducting machines, and production and machines for General Cable Superconductors at the Robinson Research Institute at Victoria university of Wellington (NZ). He is currently a Principal Engineer and specializes in the management of complex engineering projects, including customer-focused multi-disciplinary projects. He was one of the team winning the Royal Society of New Zealand Cooper Medal for the development of high temperature superconducting cables for power system applications including $1 \mathrm{MVA}$ transformer, $60 \mathrm{MW}$ hydro generator and 150 MW utility generator. He currently focusses on the application of HTS to rotating machines.

Hae-Jin Sung received B.S. and M.S. degrees in Electrical Engineering from Changwon National University, Korea in 2012 and 2014. She is currently pursuing the Ph.D. degree in the University. Her research interests are wind power system, superconducting rotating machine.

Robert A. Slade received the B.Sc. degree in Physics from Bristol University, Bristol, UK in 1986.

From 1986-1988 he completed a graduate mechanical engineering training scheme at Rolls Royce plc and specialized in gas turbine engine instrumentation. He then worked in industry developing sensors for remote cable and pipe location before joining Oxford Instruments (OI) in 1994. At OI he worked on a number of nuclear magnetic resonance (NMR) systems, notably the prototype MagTrak ${ }^{\mathrm{TM}}$ measurementwhile-drilling NMR tool in collaboration with Baker Hughes Inc., and the Hypersense $\mathrm{TM}$ dynamic nuclear polarization (DNP-NMR) system. From 2007 to 2011 he was with Siemens Magnet Technology in Eynsham, UK, where he was responsible for field stability and homogeneity of all MR imaging magnets. He is currently at the Robinson Research Institute of Victoria University, Wellington, New Zealand, where he leads the team developing high temperature superconducting magnets for magnetic resonance applications.

Mr. Slade is a member of the Institute of Physics and a Chartered Physicist. He is a named inventor for 28 patent families. 